\title{
Sabine Gerhartz-Reiter \& Cathrin Reisenauer (2020): Partizipation und Schule. Perspektiven auf Teilhabe und Mitbestimmung von Kindern und Jugendlichen.
}

\author{
Wiesbaden: Springer VS.314 Seiten, Softcover: 58,31 €/eBook: \\ 46,99€.
}

\section{Andrea Bossen}

Eingegangen: 18. Oktober 2020 / Angenommen: 20. Mai 2021 / Online publiziert: 16. Juli 2021 (C) Der/die Autor(en) 2021

Obwohl die Partizipation von Kindern und Jugendlichen $(\mathrm{KuJ})$ in der Schule sowohl in der schulischen Praxis als auch in Forschungskontexten seit Jahrzehnten als Desiderat diskutiert wird, zeigen sich gegenwärtig weiterhin Barrieren in Bezug auf die verschiedenen Akteur*innengruppen und auf unterschiedlichen Ebenen der strukturellen Ausgestaltung. Dabei ist das Recht auf Teilhabe- und Mitbestimmung seit 1989 gesetzlich in der UN-Kinderrechtskonvention Art. 12 geregelt und gehört ebenso zum guten Ton systematischer und zielgerichteter Schul- und Unterrichtsentwicklung. Der Herausgerberinnenband versammelt deutschsprachige Beiträge von Wissenschaftler*innen und Praktiker*innen überwiegend aus Österreich und der Schweiz, die zeigen, dass Partizipation in der Schule seitens der Institution und Akteur*innengruppen mit Nachdruck bejaht wird, dennoch selten in echter demokratischer Mitbestimmung als in Scheinpartizipation mündet. Wo liegen also die Herausforderungen partizipativer Prozesse in der Institution Schule? Wie nehmen die beteiligten Akteur*innen ihre Partizipationsmöglichkeiten war? Welche strukturellen Aspekte müssen bearbeitet werden, um „echte“ Teilhabe und Mitbestimmung zu ermöglichen?

Fast alle Beiträge weisen trotz unterschiedlicher Themenschwerpunkte eine ähnlich kritische Argumentationsstruktur auf, die das Spannungsfeld der Partizipation aufspannt: Die Hoffnungen und Visionen für eine gemeinschaftliche, partizipative Vorstellung von Schule, die qua institutioneller Idee und Eingebundenheit in Gesellschaft ihre Grenzen findet. Sie zeigen unterschiedliche Facetten der Partizipation, nehmen Konzepte und (politische) Modelle von Partizipation in den Blick und prüfen diese auf Verwendbarkeit in der Schule. Dabei scheint die Suche nach Handlungen,

\footnotetext{
Dr.in phil. Andrea Bossen $(\bowtie)$

Institut für Schulpädagogik und Grundschuldidaktik, Martin-Luther-Universität Halle-Wittenberg, Halle-Wittenberg, Deutschland

E-Mail: andrea.bossen@paedagogik.uni-halle.de
} 
Plänen und Strategien fast symptomatisch, u.a. auch, weil das Partizipationsverständnis der Adressat*innen sehr divers ist. Wie kann Partizipation gelingen, wenn der Begriff selbst in unterschiedlichsten Nuancen schimmert? Der Sammelband ist in vier Schwerpunktthemen gegliedert, wobei die Co-Herausgeberin Cathrin Reisenauer zunächst eine grundlegende Einführung zur Thematik gibt, unterschiedliche Begründungslinien für die Partizipation von $\mathrm{KuJ}$ in der Schule aufzeigt und ebenso konzeptionelle Darstellungen unter Einbeziehung auch internationaler Literatur diskutiert.

Im ersten Themenschwerpunkt „Partizipation und Schule als Institution“ streicht Helene J. Feichter insbesondere den Aspekt heraus, dass eine Schule, die durch Effizienz und Leistung geprägt ist, Schüler*innenpartizipation im Weg steht und daher qua Verfasstheit von Schule Partizipation einen „Kulturbruch“ (S. 29) darstellt. Maßnahmen zur Mitbestimmung sind nur erfolgsversprechend, wenn sie schulförmig sind. Die empirische Studie von Markus Ammann zeigt, dass die Haltung von Schulleitungen vor dem Hintergrund Stakeholder-orientierter Schulentwicklung und politikwissenschaftlicher Partizipationsforschung eine hohe Relevanz hat, damit Partizipation nicht zum Selbstzweck wird. Das Konzept nimmt dabei die Interessen von Anspruchsgruppen in den Blick, welche im Verhandlungsweg zusammengeführt und in je angemessener Weise ihre Berücksichtigung finden sollen. Dabei spielt die Beteiligung von Schüler*innen an der erfolgreichen Umsetzung ebenfalls eine wichtige Rolle.

Es folgt ein zweiter Themenschwerpunkt „Partizipation und Menschenrechtsbildung“, in dem Simone Danz zu einer pädagogischen Sicht auffordert. Sie versteht Verletzlichkeit und Zerbrechlichkeit als menschliche Eigenschaft und fordert, diese über einen solidarischen Umgang anerkennungsfähig zu machen. Sie betont eine Bildungsarbeit in Schule, die nur über Partizipation als eine Voraussetzung von Allgemeinbildung und Transformation der Ungleichheitsgesellschaft verwirklicht werden kann. Nadine Ulseß-Schurda berichtet aus der Schulpraxis von dem Projekt „Zeit schenken“, das im Rahmen eines neuen Wahlpflichtfachs „Social Justice Education“ angeboten und durchgeführt wurde. Das Projekt soll KuJ Erfahrungen ermöglichen, in denen sie eigenverantwortlich handeln und Selbstwirksamkeitserfahrungen auch für die Zukunft sammeln können. In den durch Frageimpulse angeleiteten Reflektionen der KuJ zeigen sich Sichtweisen auf die gesammelten Erfahrungen, teilweise werden Themen bearbeitet wie Erwachsen-Werden, der eigene „Job“ in der Welt oder Demokratieverständnisse. Der letzte Beitrag von Katrin Ehnert und Maximiliane Hädicke geht mithilfe des Modells ,Embedded Democracy“ der Frage nach, wie die Stabilitiät eines komplexen politischen Systems in eine Bildungsfrage übersetzt und in Verbindung mit Partizipation gebracht werden kann. Sie zeichnen dabei Antinomien sowohl des Partizipationsdiskurses als auch des Bildungsbegriffs nach und stellen sie in den Kontext von Demokratieförderung, was wiederum „,neue Schwierigkeiten“ evoziert. Diese Reflexion soll als Dauerthema in Bildungsinstitutionen implementiert werden, wenn sie eine produktive Grundlage für progressive pädagogische Praxis sein sollen.

„Partizipation und Inklusion“ bilden den dritten Themenschwerpunkt des Sammelbandes. Hier versammelt Susanne Thurn im Kontext inklusiver Schulentwicklung Visionen für Partizipation in ihrer Übersetzung in Erlebnismöglichkeiten, die 
wiederum in Forderungen für Inklusion gespiegelt werden. Sie beschreibt anhand von Erzählungen aus ihrem eigenen Schulalltag in der Laborschule Bielefeld, wie Barrieren der Teilhabe in spezifischen Situationen von politischen Institutionen bearbeitet werden können. Petra Flieger fragt in ihrem Beitrag nach Benachteiligungs-, Ausgrenzungs- und Diskriminierungserfahrungen bei KuJ, wie diese (mithilfe des Indexes für Inklusion) erfasst und abgebaut werden können. Unterricht soll sich am Einzelnen orientieren, daher muss die Lernumgebung angepasst werden. Diese Anpassung wird für spezifische Formen der Beeinträchtigung ausdifferenziert, wobei ein gutes Klassenklima als elementare Voraussetzung für die Akzeptanz von unterschiedlichen Lebens- und Lernbedingungen diskutiert wird. Rahel More, Ernst Kocnik und Marion Signot erörtern die Persönliche Assistenz in Österreich (PAB) als Antwort auf die Forderung, nicht über Menschen mit Behinderung und ihre Teilhabe zu entscheiden, sondern diese in die Planung und Umsetzung von barrierefreiem Lernen einzubeziehen. Das partizipative Forschungsprojekt „Frauen mit Lernschwierigkeiten zwischen Selbst- und Fremdbestimmung" leitet angemessene Unterstützung für Selbststimmung als Voraussetzung für gesellschaftliche Partizipation ab, indem etablierte defizitorientierte Klassifikationen durch neue partizipative und bedarfsorientierte Handlungsstrategien ersetzt werden.

Im vierten Themenschwerpunkt wird „Partizipation und ihre Bedeutung für Schüler*innen“ in den Mittelpunkt gestellt. Dazu werden im ersten Beitrag Texte von Schüler*innen gesammelt, in denen sie ihre Erfahrungen, Möglichkeiten und Anliegen bezüglich der Partizipation in der Schule artikulieren. Insgesamt sind die Partizipationsdesiderate breit gefächert, von hoher Zufriedenheit mit der aktuellen Situation bis hin zu großem Unmut. Dabei werden insbesondere die gegenseitige Akzeptanz von Lehrperson und Schüler*innen, die Mitbestimmung von Unterrichtsinhalten sowie eine allgemeine Partizipation thematisiert. Schüler*innenseits werden ferner konkrete Vorschläge gemacht, was geändert werden müsste, um eine höhere Partizipation zu erreichen. Daniela Müller-Kuhn, Julia Häbig, Enikö Zala-Mezö, Nina-Cathrin Strauss und Pascale Herzig untersuchen in einer Studie, wie Schüler*innen in der Mittel- und Oberstufe Partizipation wahrnehmen und wie sich dies von einer zur nächsten Stufe verändert. Mithilfe eines Partizipationsmodells mit Fokus auf Unterricht zeigen die Daten, dass die Lehrpersonen und Schüler*innen zu beiden Messzeitpunkten eher wenig Partizipation im Unterricht hinsichtlich organisatorischer und inhaltlicher Offenheit wahrnehmen, wobei Schüler*innen nochmal signifikant geringere Möglichkeiten sehen. Im Zusammenhang mit der (Un-)Zufriedenheit wird u. a. die Schulkultur diskutiert, sodass Partizipation als schulweites und nicht individuelles Thema der Lehrperson betrachtet werden soll. In einer weiteren Studie untersuchen Manuela Gamsjäger und Daniela Wetzelhütter, inwieweit Schüler*innen Einflussnahme und Scheinpartizipation erleben und welche Konsequenzen das für das Engagement der Schüler*innen hat. Das Spektrum der erfahrenen Beteiligung reicht vom Erleben eines Einflusses auf wichtige Entscheidungen bis zur bloßen Information darüber oder gar vorgetäuschter Partizipation. Klassenspezifische Merkmale haben dabei großen Einfluss auf die Partizipationsintensitäten; so geht beispielsweise ein positives Klassenklima seltener mit unzureichender und symbolischer Partizipation einher. Im letzten Beitrag des Themenschwerpunktes untersuchen Daniela Martinek und Matteo Carmignola, wie aufgrund der Einführung 
von Bildungsstandards und der damit verbundenen evidenzbasierten Steuerungslogik und Ergebnisorientierung der Schule Lehrer*innen bezüglich ihrer Unterrichtsqualität unter Druck geraten. Dieser wird auch für Schüler*innen, insbesondere in Testsituationen und draus resultierenden Versagensängsten, spürbar, was wiederum schulische Bildung eher boykottiert als gute Lernergebnisse fördert. Die Ergebnisse zeigen, dass es nicht nur einiger Maßnahmen bedarf, um dem Wunsch nach Partizipation der Schüler*innen gerecht zu werden, sondern Schul- und Unterrichtsentwicklung von Anfang an eine aktive Einbindung der Schüler*innen einplanen muss.

Im Themenschwerpunkt „Partizipation und Bildungswege“ nimmt Sabine Gerhartz-Reiter Normen, Barrieren und Machtverhältnisse in den Blick, die bearbeitet werden müssten, um eine ,echte“ Beteiligung an Entscheidungen in Bezug auf Bildungsprozessen von KuJ zu ermöglichen. Sowohl strukturelle Rahmenbedingungen im österreichischen Schulsystem als auch eine mangelnde Teilhabe führe zu Barrieren für selbstständige Bildungsverläufe, die wiederum eng verwoben sind mit der Identitätsentwicklung von KuJ. Allerdings sei im pädagogischen Kontext Partizipation immer eine Partizipation von Ungleichen, sodass die Entwicklung einer Entscheidungskompetenz zur Voraussetzung wird, um auch bildungspolitisch mitbestimmen zu können. Marie Gitschthaler, Franziska Lessky und Erna Nairz-Wirth untersuchen in einer Evaluationsstudie an berufsbildenden Schulen, inwiefern das „Student Engagement“ (SE) durch selbstgesteuertes Lernen begünstigt wird und einem Dropout entgegenwirken kann. Dabei zeigen Lernbüro-Schüler*innen (LS) ein signifikant höheres SE als die Vergleichsgruppe und eine niedrigere Dropout Rate. LS empfinden ihre Zeit in Schule weniger als verschwendet. Sie bewerten die Autonomie bezogen auf Lernprozess als auch den hohen Einfluss des Engagements durch Beziehungsdimension als positiv. Allerdings gibt es auch im Zeitverlauf einen leicht signifikanten Rückgang des Engagements in beiden Gruppen, was über die gestiegenen Anforderungen und dadurch evozierten Unsicherheiten legitimiert wird. Im letzten Beitrag stellt Eveline Christof Partizipation als bedeutsames Thema der Lehrer*innenbildung heraus. In einer Fragebogenstudie mit Lehramtsstudierenden werden diese nach ihren Sichtweisen/Möglichkeiten/Herausforderungen und Grenzen von und zu Partizipation befragt. Das Ergebnis zeigt, dass Partizipation ein positiv besetzter Begriff ist, der aus Studierendenperspektive in Schule aufgrund struktureller Gegebenheiten seine Ambivalenzen findet. Die Autorin betont, dass eine Reflexion der berufsbezogenen Überzeugungen in der Aus- und Weiterbildung zentral ist, um nicht nur eine Veränderung zu ermöglichen, sondern auch Zusammenhänge zu anderen Themen wie individuelle Förderung, Leistungsbeurteilung oder Autorität zu diskutieren.

\section{Kritische Würdigung}

Über den Band hinweg wird deutlich, dass die Forderung nach (Mit-)Bestimmung ein politischer Aufruf ist, deren Legitimation ähnliche Begründungslinien aufweist. Interessant ist, dass es gleichwohl unterschiedliche Ausgestaltungen der Teilhabe gibt, die einerseits durch machtvolle strukturelle Barrieren konturiert werden, 
anderseits durch die Denkmuster und Handlungen beteiligter Akteur*innen (Lehrer*innen und Schüler*innen). Die Beteiligten selbst artikulieren diverse Partizipationsverständnisse von weitreichender Mitbestimmung bis hin zur Aushandlung und Ermöglichung von Mitsprache. Die Betonung von Partizipation als diskursiven, spannungsreichen Prozess ist elementar, um eine echte Eingebundenheit von $\mathrm{KuJ}$ in kollektive Entscheidungsprozesse zu erwirken. Die Schaffung von Entscheidungsräumen muss zu einer verbindlichen Einflussnahme führen. Dieser Prozess ist allerdings voraussetzungsvoll und bedarf sowohl des Aufbruchs struktureller Rahmenbedingungen als auch Kompetenzen, die in Schule gefördert werden sollten (Selbstständigkeit, Entscheidungskompetenzen, usw.). Offen bleibt, wie Partizipation in Bezug zum Bildungsbegriff gesetzt werden kann, der in einigen Beiträgen eher unvermittelt hinzukommt. Zudem bleibt die Frage unbearbeitet, inwiefern Partizipation zwischen schulischer Programmatik und Ideal/Desiderat einer demokratischen Gesellschaft laviert und in welchem Verhältnis ein institutionelles Verständnis von Partizipation zu einem gemeinschaftlichen Verständnis im Sinne einer Schulgemeinschaft steht. Die zuweilen als überfordert dargestellte Praxis ist auch Ausdrucksform antinomischer Ansprüche. Dies spiegelt sich ebenso in einem Vokabular wieder, welches nicht immer passförmig mit einer partizipativen Vorstellung von Schule und Gesellschaft ist. So müsste bspw. die Konstruktion relevanter Differenzdimensionen (,Umgang mit Anderen“) weiter herausgearbeitet oder auch das Nutzenprinzip reflektiert werden, damit Partizipation nicht als Mittel zum Zweck (z.B. Zwang zur Verantwortung, Verweis auf den Gebrauch in einer unbestimmten Zukunft) fungiert, sondern als Wert an sich erkennbar wird.

Funding Open Access funding enabled and organized by Projekt DEAL.

Open Access Dieser Artikel wird unter der Creative Commons Namensnennung 4.0 International Lizenz veröffentlicht, welche die Nutzung, Vervielfältigung, Bearbeitung, Verbreitung und Wiedergabe in jeglichem Medium und Format erlaubt, sofern Sie den/die ursprünglichen Autor(en) und die Quelle ordnungsgemäß nennen, einen Link zur Creative Commons Lizenz beifügen und angeben, ob Änderungen vorgenommen wurden.

Die in diesem Artikel enthaltenen Bilder und sonstiges Drittmaterial unterliegen ebenfalls der genannten Creative Commons Lizenz, sofern sich aus der Abbildungslegende nichts anderes ergibt. Sofern das betreffende Material nicht unter der genannten Creative Commons Lizenz steht und die betreffende Handlung nicht nach gesetzlichen Vorschriften erlaubt ist, ist für die oben aufgeführten Weiterverwendungen des Materials die Einwilligung des jeweiligen Rechteinhabers einzuholen.

Weitere Details zur Lizenz entnehmen Sie bitte der Lizenzinformation auf http://creativecommons.org/ licenses/by/4.0/deed.de. 